\title{
Tuning Frequencies Across Leads with Interleaving Deep Brain Stimulation to Treat Asymmetric Tremor in Parkinson's Disease
}

\author{
Massimo Marano (iD, Francesco Motolese, Daniele Marruzzo, \\ Riccardo Antonio Ricciuti, Vincenzo Di Lazzaro, Manuela Pilleri
}

Keywords: Subthalamic nucleus, Internal capsule, Troubleshooting, Motor fluctuations, Neuromodulation

doi:10.1017/cjn.2020.64

Can J Neurol Sci. 2020; 47: 557-559

The subthalamic nucleus deep brain stimulation (STN-DBS) is an efficacious treatment for advanced Parkinson's disease (PD). Interleaving stimulation (ILS) is a DBS programming modality, which is adopted when conventional configurations (e.g. monopolar or bipolar) fail. Although the therapeutic mechanism is not fully understood, ILS aims (i) to reduce side effects and/or (ii) to stimulate different therapeutic sites within STN or nearby areas in order to control specific symptoms. ${ }^{1}$ For instance, through the contemporary stimulation of the STN and of a more dorsal contact, it is possible to target the pallidofugal tract to relieve dyskinesias ${ }^{2}$ or the zona incerta to treat tremor. ${ }^{3}$ Each stimulating contact could be programmed with an individual combination of amplitude and pulse width, but with a single frequency value that could not be higher than $125 \mathrm{~Hz}^{4}$ Hence, ILS has been generally considered less effective on tremor, given that the latter usually improves at higher frequencies. ${ }^{4}$ Herein, we present a trick to control asymmetric PD tremors overcoming two main ILS limitations, namely, the inability (i) to generate frequencies higher than $125 \mathrm{~Hz}$ focused on a cathode and (ii) to program independent frequencies across leads. 5

A 54-year-old woman with a 12-year history of PD and a modified Hoehn and Yahr score of 3 received a STN-DBS because of motor fluctuations, characterized by sudden and unpredictable offs and disabling peak-dose coreoathethoid dyskinesias, which were no more treatable with oral therapies. Besides, the patient also reported rest tremor of moderate to severe entity, located mainly on her right body side, with a suboptimal response to levodopa. The preoperative levodopa equivalency daily dose (LEDD) was $1154 \mathrm{mg}$. She received bilateral STN-DBS (Activa-PC, Medtronic), leading to a good control of motor symptoms, except for the right-side tremor (on and off medications). Left STN voltage was increased with improvement of tremor at the cost of side effects (i.e. right facial pulling) on monopolar and bipolar configurations. Hence, keeping left STN voltages below the threshold of side effects, frequencies were raised up to $220 \mathrm{~Hz}$ with a complete control of right-side tremor but with the concurrent onset of a facial pulling on the left side. Any attempt to lower the right STN voltage in order to reduce the new stimulation side effect caused a loss of therapeutic benefit. After a further unsuccessful attempt to control tremor stimulating the more dorsal and the more therapeutic contacts with ILS (i.e. zona incerta plus STN stimulation), ${ }^{5}$ a definitive and lasting symptom control was achieved by adopting two interleaved bipolar programs sharing a cathode, pulsing at $250 \mathrm{~Hz}$ (as the result of the overlapping $125 \mathrm{~Hz}$ fields) in the left lead - i.e., contralateral to the worst trembling body side (Figure 1, Supplementary Table). The LEDD was reduced to $540 \mathrm{mg}$, with an 18 -month follow-up.

ILS is an advanced programming technique, which is adopted to sculpt the electrical field with the drawback of a quicker battery drain. ${ }^{1,5}$ Each electrode could host a couple of virtual leads with a frequency ceiling of $125 \mathrm{~Hz}$, which was imposed by the manufacturer in order to avoid excessive electrical charge density (e.g. occurring over $250 \mathrm{~Hz}$ ). ${ }^{1,5}$ On a clinical point of view, higher frequencies (i.e. $>130 \mathrm{~Hz}$ ) are often required to achieve a better control of tremor. ${ }^{4}$ Therefore, the possibility to select different frequencies per lead (e.g. higher frequencies contralateral to the trembling body side) would be of help in treating asymmetric frequency-sensitive tremors. This is possible with a unilateral ILS, which is able to increase frequencies of the small region that is included in the overlap area of two close interleaved fields (i.e. running with a phase delay) ${ }^{6}$ However, the proposed ILS setting let the entire left cathode pulsing at a higher frequency

From the Unit of Neurology, Neurophysiology and Neurobiology, Department of Medicine, Campus Bio-Medico of Rome University, Rome, Italy (MM, FM, VDL); Neurosurgery Unit, Department of Neurosciences, Belcolle Hospital, ASL of Viterbo, Viterbo, Italy (DM, RAR); and Department of Neurology, Villa Margherita Clinic, Arcugnano, Vicenza, Italy (MP)

Received February 22, 2020. Final Revisions Submitted March 11, 2020. Date of Acceptance March 24, 2020.

Correspondence to: Massimo Marano, Unit of Neurology, Neurophysiology and Neurobiology, Department of Medicine, University Campus Bio-Medico of Rome, Viale Alvaro del Portillo 200, 00128, Rome, Italy. Email: m.marano@unicampus.it, masmarano@gmail.com 


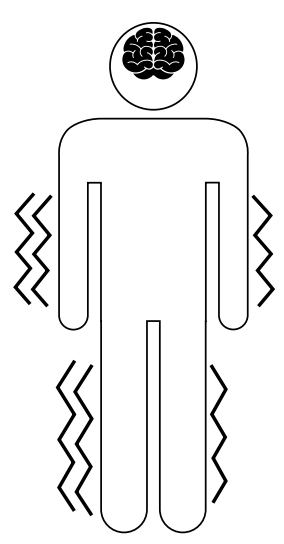

Stimulator OFF

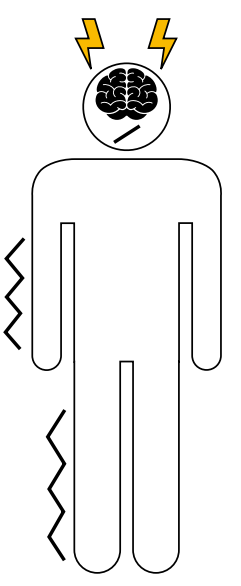

C+1-: 3.4, 60, 130

C+9-: 2.5, 60, 130

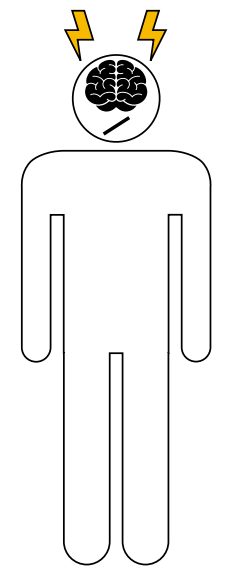

C+1-: 3.4, 60, 180

C+9-: $2.5,60,180$
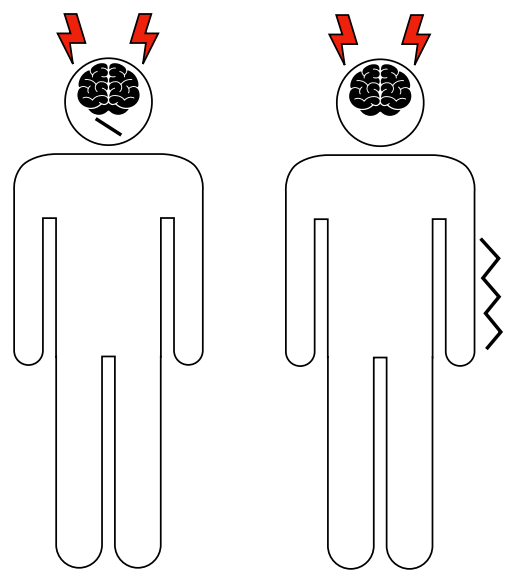

$C+1-: 3.2,60,220$

$C+1-: 3.2,60,220$

C+9-: 2.5, 60, 220

C+9-: 2.0, 60, 220

$0+1-: 3.2,60,125^{*}$

$C+9-:$ 2.5, 60, 125

\begin{tabular}{|lll}
\hline & Tremor & High frequency \\
3 & Stimulation & Interleaving
\end{tabular}

Figure 1: Graphical representation of the patient programming sessions. It was not possible to obtain a full symptomatic control with standard programming techniques (single yellow thunderbolt) due to the presence of stimulation-induced side effects (face oblique line pending to the facial pulling side) on with or without incomplete tremor (wavy line) suppression. Stimulating at higher frequencies (red thunderbolts) was effective on tremor, at constant values of pulse amplitude and width. However, this leads to the appearance of stimulation-induced side effects on the least affected body side. ILS (double-yellow thunderbolt) allowed to adopt different stimulation settings across leads, in particular it was possible to adopt asymmetric frequencies with a full control of tremor on the most affected body side, without adverse effects.

than the right one, with a consecutive robust tremor improvement. Of relevance, two interleaved programs could share an entire cathode only if the latter is included into a couple of bipolar configurations (e.g. $2+1-$ and $0+1-$ ).

The approach we described here would be a further resource in the management of asymmetric PD tremor. Moreover, it could also be speculated that the use of ILS and of higher frequencies would be of help in reducing the exaggerated oscillatory activity pattern in the beta band, a well-known finding in tremordominant PD patients. ${ }^{7}$

Finally, more studies are warranted to better understand the mechanism behind the ILS effect and how it could help clinicians in "tailoring" DBS therapy for their patients.

\section{FUNDING}

This research did not receive any specific grant from funding agencies in the public, commercial, or not-for-profit sectors.

\section{DiscLOSURES}

Dr Massimo Marano received speaker honoraria and consultancies by AbbVie and Allergan, research grant by "Alberto Sordi Foundation"; Dr Francesco Motolese reports no disclosures; Dr Daniele Marruzzo reports no disclosures; Dr Riccardo Antonio Ricciuti reports no disclosures; Prof. Vincenzo Di Lazzaro reports no disclosures; $\operatorname{Dr}$ Manuela Pilleri consultancies by Boston Scientific, Brainlab, speaker's honoraria for UCB, AbbVie, Zambon.

\section{Statement of Authorship}

MM, FM, DM clinical data collection and drafting of the manuscript; RAR, VDL, and MP drafting the article and revising it critically for important intellectual content, final approval of the version to be submitted.

\section{Ethics Approval and Consent to Participate}

The authors declare that ethics approval was not required for this case report that is conducted in accordance with the declaration of Helsinki.

\section{SupPlementary Material}

To view supplementary material for this article, please visit https://doi.org/10.1017/cjn.2020.64.

\section{REFERENCES}

1. Miocinovic S, Khemani P, Whiddon R, et al. Outcomes, management, and potential mechanisms of interleaving deep brain stimulation settings. Parkinsonism Relat Disord. 2014;20(12):1434-7. doi: 10.1016/j.parkreldis.2014.10.011.

2. Aquino CC, Duffley G, Hedges DM, et al. Interleaved deep brain stimulation for dyskinesia management in Parkinson's disease. Mov Disord. 2019;34(11):1722-7. doi: 10.1002/mds.27839.

3. Kern DS, Picillo M, Thompson JA, et al. Interleaving stimulation in Parkinson's disease, tremor, and dystonia. Stereotact Funct Neurosurg. 2018;96(6):379-91. doi: 10.1159/000494983.

4. Moro E, Esselink RJA, Xie J, Hommel M, Benabid AL, Pollak P. The impact on Parkinson's disease of electrical parameter settings in STN stimulation. Neurology. 2002;59:706-13. 
5. Amon A, Alesch F. Systems for deep brain stimulation: review of technical features. J Neural Transm (Vienna). 2017;124(9): 1083-91. doi: 10.1007/s00702-017-1751-6.

6. França C, Barbosa ER, Iglesio R, Teixeira MJ, Cury RG. Interleaving stimulation in Parkinson disease: interesting to whom? World
Neurosurg. 2019;130:e786-93. doi: 10.1016/j.wneu.2019.06. 223. Epub 2019 Jul 8. PubMed PMID: 31295615.

7. Cagnan H, Duff EP, Brown P. The relative phases of basal ganglia activities dynamically shape effective connectivity in Parkinson's disease. Brain. 2015;138(Pt 6):1667-78. doi: 10.1093/brain/awv093. 